University of Nebraska - Lincoln

DigitalCommons@University of Nebraska - Lincoln

\title{
8-4-1997
}

\section{Scanning Motions of an Atomic Force Microscope Tip in Water}

\author{
Kenichiro Koga \\ University of Nebraska-Lincoln, koga@cc.okayama-u.ac.jp \\ Xiao Cheng Zeng \\ University of Nebraska-Lincoln, xzeng1@unl.edu
}

Follow this and additional works at: https://digitalcommons.unl.edu/chemzeng

Part of the Chemistry Commons

Koga, Kenichiro and Zeng, Xiao Cheng, "Scanning Motions of an Atomic Force Microscope Tip in Water" (1997). Xiao Cheng Zeng Publications. 68.

https://digitalcommons.unl.edu/chemzeng/68

This Article is brought to you for free and open access by the Published Research - Department of Chemistry at DigitalCommons@University of Nebraska - Lincoln. It has been accepted for inclusion in Xiao Cheng Zeng Publications by an authorized administrator of DigitalCommons@University of Nebraska - Lincoln. 


\title{
Scanning Motions of an Atomic Force Microscope Tip in Water
}

\author{
Kenichiro Koga and X. C. Zeng \\ Chemistry Department and Center for Materials Research and Analysis, University of Nebraska-Lincoln, Lincoln, Nebraska 68588
}

(Received 23 April 1997)

\begin{abstract}
Integral equation techniques are used to study scanning motions of a single-atom tip of the atomic force microscope (AFM) over a rigid, hydrophobic monolayer substrate in water. The calculated force curve is found to be oscillatory, in agreement with recent AFM experiments, which can lead to multiple scanning trajectories for the tip under a constant load. The unique trajectory along which the system is thermodynamically stable is revealed. This study shows that the tip may take a hopping motion over a defect-free substrate due to layering of water molecules between the tip and substrate. [S0031-9007(97)03741-1]
\end{abstract}

PACS numbers: 61.16.Ch, 61.20.Gy, 62.20.Qp, 68.45.-v

Since its invention the atomic force microscope (AFM) [1] has permitted the high resolution imaging of surfaces at nanometer and subnanometer scales. Recently, AFM has been taken in liquid environments [2-8] to achieve, for example, true atomic resolution [2] or as a tool for biological applications [5-8]. There are several advantages to performing measurements in liquids including elimination of capillary forces and provision of native environments for biological samples.

O'Shea, Welland, and Rayment [4] have used AFM to investigate force curves (normal forces on the tip versus tip-substrate distance) of systems immersed in liquids. Under octamethylcyclotetrasiloxane liquid they observed a few oscillations in the force curves, similar to those observed using the surface force apparatus $[9,10]$, where the oscillations in the force curves were due to layering of the liquid molecules between two parallel solid surfaces. Cleveland, Schäffer, and Hansma [5] have measured forces between AFM tips and hydrophilic cleavage planes of the ionic crystal of calcite and barite in water. The forces were also found to be oscillatory. Moreover, recent computer simulations [11,12] have also confirmed the oscillatory forces between AFM tips and solid surfaces in liquids.

Scanning motions of AFM tips in air over various surfaces have been the subject of several reviews [13,14]. To our knowledge, scanning motions of AFM tips in liquids have not yet been studied theoretically. The focus of this paper is to investigate distinctive features of scanning motions of an AFM tip over a rigid monolayer substrate in water. Because forces between the tip and substrate, when both are immersed in water, are oscillatory, multiple scanning trajectories for the tip under a constant load may arise. On most of these trajectories, however, the system is at metastable or unstable equilibrium. Analysis of the total potential of mean force reveals a unique scanning trajectory along which the system is thermodynamically stable. This trajectory shows that the tip can take a hopping motion when it scans over a defect-free substrate, because of layering of the water molecules between the tip and the substrate.
We present here the results of force curves and scanning trajectories of an AFM tip over the monolayer substrate in water. We used the integral equation technique of statistical mechanics [15]. For simplicity, we have restricted our study to the system including a single-atom tip and a rigid monolayer substrate consisting of $N_{s}$ atoms fixed at hexagonal close-packed lattice sites (Fig. 1), both immersed in water ( $N_{w}$ molecules). The system is taken to be as large as a liquid cell in the real experiment (which is impractical to simulate by using conventional methods such as molecular dynamics or Monte Carlo). The substrate lattice sites are placed at the $z=0$ plane and the tip is placed in the positive $z$ space; the $z$ coordinate of the tip atom is then considered as the tip height. Temperature $T$ and volume $V$ of the system as well as $N_{w}$ are held constant. In addition, the lateral position $(x, y)$ and the tip height $z$ or an external normal force $-F_{z}^{\text {ext }}$ (the load) acting on the tip are controlled as external parameters. The latter case corresponds to a constant load $\left(-F_{z}^{\text {ext }}\right)$ condition for the tip. An equilibrium state of the system is completely specified by $N_{w}, V, T, x, y$, and $z$ or by $N_{w}$, $V, T, x, y$, and $F_{z}^{\mathrm{ext}}$.

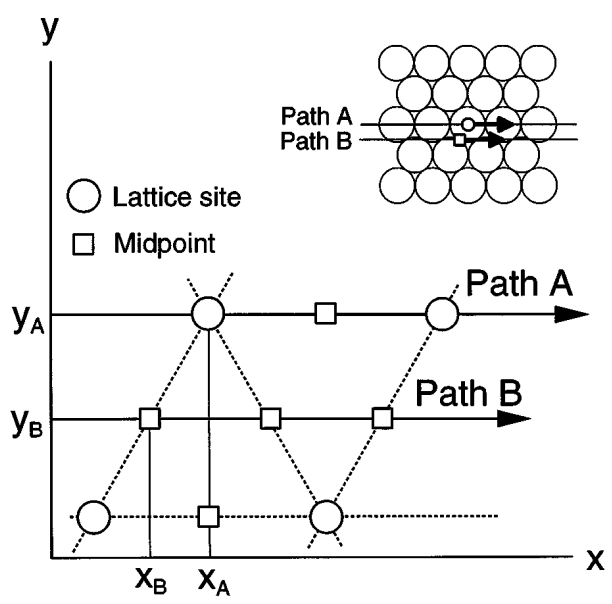

FIG. 1. A portion of the hexagonal close-packed substrate and two pathways of the tip. Coordinates $\left(x_{A}, y_{A}\right)$ and $\left(x_{B}, y_{B}\right)$ are the initial positions of the tip on paths $A$ and $B$. 
The tip-substrate interaction in vacuum, $U^{\mathrm{vac}}(\mathbf{r})$, is taken to be a pairwise sum, $U^{\mathrm{vac}}(\mathbf{r})=\sum_{j=1}^{N_{s}} u_{t s}\left(r_{j}\right)$, where $u_{t s}$ is the Lennard-Jones (LJ) potential: $u_{t s}(r)=$ $4 \epsilon\left[(\sigma / r)^{12}-(\sigma / r)^{6}\right]$ and $r_{j}$ denotes the distance between the tip atom and $j$ th atom of the substrate; $\epsilon$ and $\sigma$ are the LJ energy and size parameters. We used parameters for a site-site model of naphthalene [16] in the calculations, $\epsilon_{s} / k=67.1 \mathrm{~K}, \sigma_{s}=3.395 \AA$, where $k_{B}$ denotes the Boltzmann constant. The molecular potential of water is described by a slightly modified TIPS sitesite interaction potential [17]. The water-tip and watersubstrate intermolecular interactions are described by LJ potentials with the parameters determined by combining rules, $\epsilon_{w s}=\sqrt{\boldsymbol{\epsilon}_{w} \boldsymbol{\epsilon}_{s}}$ and $\sigma_{w s}=\left(\sigma_{w}+\sigma_{s}\right) / 2$. The lattice constant $l_{a}$ of the monolayer substrate is set to be $2^{1 / 6} \sigma_{s}$. Water temperature is set at $298.15 \mathrm{~K}$ and density at $1 \mathrm{~g} / \mathrm{cm}^{3}$.

In the actual AFM experiments, a typical time scale for a tip scanning over one nanometer is of the order of one millisecond, which is much longer than the time scale required for the attainment of thermodynamic equilibrium of liquids. Therefore we consider motion of the tip as a quasistatic process under a constant $N_{w} V T$ condition, that is, as a succession of equilibrium states, each state being specified by $x, y$, and $z$ or $x, y$, and $F_{z}^{\text {ext }}$. Note that during the scanning process all the thermodynamic properties of bulk water are kept constant, including chemical potential and pressure. At each equilibrium state, the tip experiences an intermolecular force $\mathbf{F}^{\text {int }}=$ $\mathbf{F}^{\mathrm{vac}}+\mathbf{F}^{\text {solv }}$, where $\mathbf{F}^{\mathrm{vac}}$ and $\mathbf{F}^{\text {solv }}$ are forces due to the tip-substrate direct interaction in vacuum and the solventinduced indirect interaction, respectively. $\mathbf{F}^{\text {int }}(\mathbf{r})$, given by $\mathbf{F}^{\text {int }}(\mathbf{r})=-\nabla U^{\text {int }}(\mathbf{r})$, is the mean force acting on the tip fixed at $\mathbf{r}=(x, y, z)$, that is, a force canonically averaged over the configurations of the solvent molecules. $U^{\text {int }}$ is the potential of mean force which consists of two terms:

$$
U^{\text {int }}(\mathbf{r})=U^{\mathrm{vac}}(\mathbf{r})+U^{\mathrm{solv}}(\mathbf{r}),
$$

where $U^{\text {solv }}$ is the solvent-induced potential contribution.

We determine $U^{\operatorname{solv}}(\mathbf{r})$ as a function of the tip's lateral position $(x, y)$ and height $z$ by use of the supermolecule approach $[16,18]$ based on the extended reference interaction site model (RISM) integral equation technique [19]. Details of this approach have been given elsewhere $[16,18,20]$. The supermolecule approach allows one to evaluate the solvent-induced potential of mean force between two molecules without invoking the detailed dynamics of the solvent molecules, thereby considerably reducing the computational effort. Semiquantitative agreement with computer simulations has been obtained $[16,18]$. Here we have extended the supermolecule approach to treat a two-dimensional monolayer substrate consisting of a large number of atoms by introducing a two-dimensional periodicity in the $x$ and $y$ directions. Similar extension was made by other work- ers to study polymers in solvents [21]. Specifically, the periodic unit consists of the tip and a rectangular section of the substrate (containing 132 atoms) with side length $L_{x}=11 l_{a}=41.92 \AA$ and $L_{y}=6 \sqrt{3} l_{a}=$ $39.60 \AA[22,23]$. The tip is moved laterally along a straight line referred to here as a path. Two paths are examined (see Fig. 1): Path $A$ is defined by $y=y_{A}$ and path $B$ by $y=y_{B}$. The solvent-induced interaction $U^{\text {solv }}$ is calculated at $21 \times 21$ grid points covering a section between $x_{\alpha}$ and $x_{\alpha}+l_{a} / 2$ and between $z_{l}$ and $z_{h}$ on a $x z$ plane at $y=y_{\alpha}$, where $\alpha=A$ or $B, z_{l}=0.75 \sigma_{w s}$, and $z_{h}=2 \sigma_{w s} ; U^{\text {solv }}$ at finer grid points is then evaluated using an interpolation method.

Figure 2 displays the calculated force curves, i.e., $F_{z}^{\text {int }}$ versus the tip height $z$. Clearly, the force curves are oscillatory. This feature is generic regardless of the lateral position of the tip above the lattice [see Figs. 2(a) and 2(b)]. The atomic-scale force oscillations are attributed to the packing of liquid molecules near solid surfaces [9]. Here we attempt to reveal how the force oscillations alter scanning motions of the tip.

Since we have assumed that the AFM tip scans quasistatically the total force on the tip should be zero. The height of the tip at equilibrium, $z^{\mathrm{eq}}$, at any lateral position $(x, y)$, can be determined via the force balance equation

$$
F_{z}^{\text {int }}(z)+F_{z}^{\text {ext }}=0 .
$$

If an iso-force $\left(-F_{z}^{\text {ext }}\right)$ horizontal line is drawn in Fig. 2 one may find multiple intersections of the iso-force line with the force curve $\left[F_{z}^{\text {int }}(z)\right]$. In other

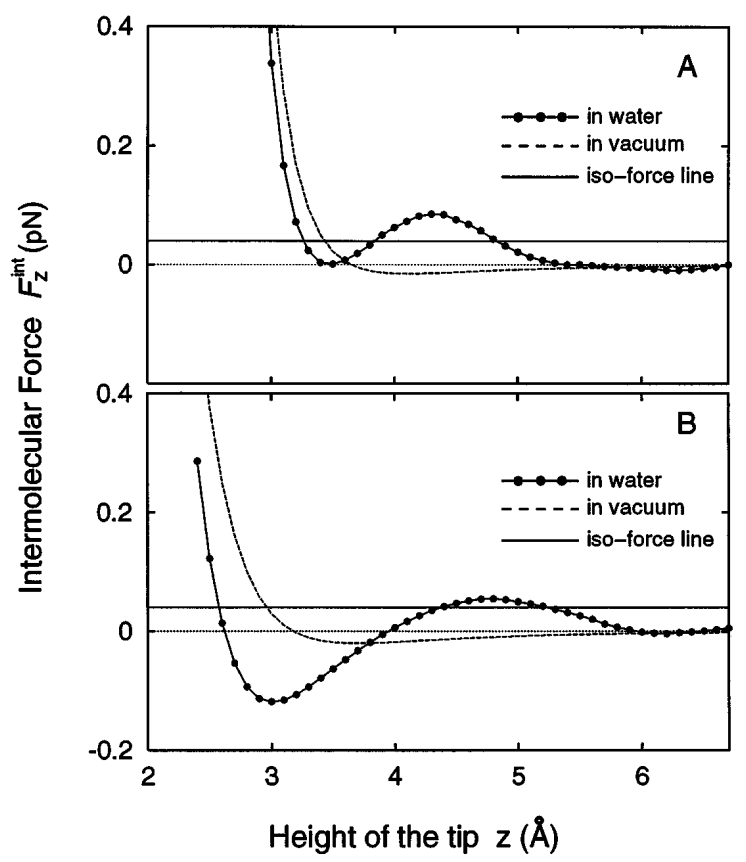

FIG. 2. Force curves $F_{z}^{\text {int }}$ of the single-atom tip as a function of the tip height $z$ above the substrate: (a) on top of a lattice site and (b) on top of a midpoint. The horizontal solid line denotes an isoforce line $(40 \mathrm{pN})$. 
words, multiple values $\left\{z_{i}^{\mathrm{eq}}\right\}$ can satisfy Eq. (2) because of the force oscillations. Consequently, there exist a number of trajectories for the tip under the same load. In contrast, there exists only one trajectory for the tip in vacuum [24].

Although a number of equilibrium states of the system, characterized by $\left\{z_{i}^{\mathrm{eq}}\right\}$, are allowed for a given load, only one of them is thermodynamically stable and the others are either metastable or unstable. We present here a simple procedure to find out the trajectory along which the system is at the stable equilibrium state. First, we divide the equilibrium states into two groups by inspecting the slope of the force curve $\partial F_{z}^{\mathrm{int}} / \partial z$ at every $z_{i}$. States in the first group which have positive slopes are unstable because any deviation from $z_{i}^{\mathrm{eq}}$ is accompanied by a net force $\left(F^{\text {tot }}=F^{\text {int }}+F^{\text {ext }}\right)$ which causes more deviation; states in the second group which have negative slopes are either stable or metastable because the net force restores the system to the original equilibrium state. Next, to find out the stable state among states in the second group we calculate the total potential of mean force, $U^{\text {tot }}(\mathbf{r})=U^{\text {int }}(\mathbf{r})-F_{z}^{\text {ext }} z$, whose derivative with respect to $z$ gives rise to $F_{z}^{\text {tot }}$. The stable equilibrium state, characterized by $z_{s}^{\mathrm{eq}}$, possesses the lowest total potential of mean force. The contour of $z_{s}^{\mathrm{eq}}$ along a given path depicts the unique scanning trajectory (hereafter we call it the stable trajectory) for the tip under the constant load.

Figures 3(a)-3(c) show the stable trajectories along path $A$ (Fig. 1) under three different loads [25]. The dotted curves delineate all possible metastable and unstable trajectories of the tip along path $A$. Under the smallest load $\left(-F_{z}^{\text {ext }}=10 \mathrm{pN}\right)$ three continuous trajectories are found [denoted by 1, 2, and 3 in Figs. 3(a) and 3 (b)]. Along trajectory 1 the tip is always in contact with the substrate $(z \sim 3 \AA)$ while along trajectory 3 the tip is separated from the substrate by one hydration layer $(z \sim 5 \AA)$. Since the tip is unstable on trajectory 2 the stable trajectory can only be portions of either trajectory 1 or 3 , depending upon the tip's position. We found trajectory 3 is more stable when the $x$ coordinate of tip is within $0.3 l_{a}$ from a lattice site while trajectory 1 is more stable otherwise. This is because when the tip is on top of a lattice site the total potential of mean force is higher on trajectory 1 than that on trajectory 3 . As the tip moves away from the lattice site the total potential of mean force gradually decreases on trajectory 1 and eventually becomes lower than that on trajectory 3 . Here the solvent-induced potential $U^{\text {solv }}$ plays a key role. When the tip is on top of a lattice site $U^{\text {solv }}$ is about $2.4 k_{B} T$ on trajectory 1 , which is higher than that on trajectory 3 . However, when the tip is on top of the midpoint $U^{\text {solv }}$ is about $1.5 k_{B} T$ on trajectory 1 , which is lower than that on trajectory 3 . Consequently, the stable trajectory exhibits a stepwise feature, indicating that the tip may hop back and forth between trajectories 1 to 3 during the quasistatic scan along path $A$ [26]. If the load is increased, more portions of trajec-

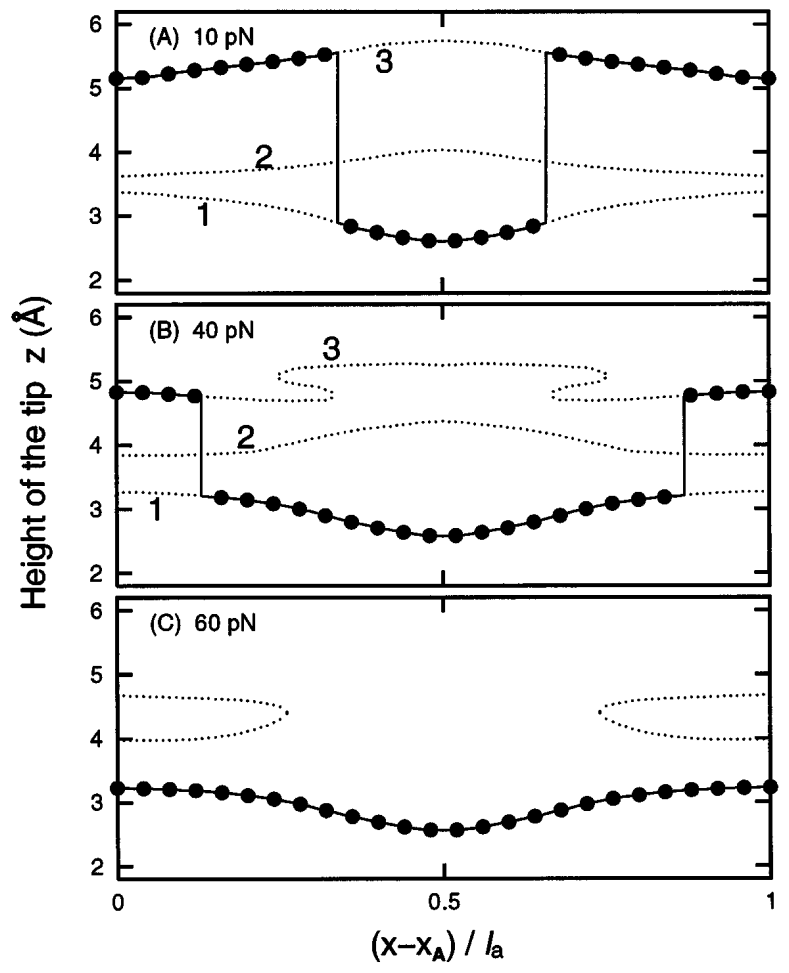

FIG. 3. Solid lines (with heavy dots) delineate the stable trajectory of the tip when moving along path $A$ under three different constant loads: (a) $10 \mathrm{pN}$, (b) $40 \mathrm{pN}$, and (c) $60 \mathrm{pN}$. Light dotted lines delineate all possible metastable and unstable trajectories of the tip. $l_{a}$ is the lattice constant of the monolayer substrate.

tory 1 become the stable trajectory [Fig. 3(b)]; and under very high load the entire trajectory 1 becomes the stable trajectory [Fig. 3(c)]. It is of interest to calculate the potential barrier $\Delta^{\ddagger} U^{\text {tot }}$ separating two trajectories 1 and 3 . Note that trajectory 2 corresponds to the ridge of the potential barrier. We find the barrier at the coordinate where the tip jumps is $1.2 k_{B} T$ under the load $10 \mathrm{pN}$ and $0.6 k_{B} T$ under the load $40 \mathrm{pN}$, which is comparable to the thermal energy. A similar conclusion has been made from experiment [5].

Figure 4 displays the stable trajectories along path $B$ under three loads, which look quite different from those along path $A$. Although three trajectories are also found under the lowest as well as the medium loads [Figs. 4(a) and 4(b)], trajectory 1 is always the stable trajectory throughout path $B$. Under the higher load no multiple trajectories are found; the sole trajectory is the stable trajectory [Fig. 4(c)] on which the tip is always in close contact with the substrate.

In this study, we have revealed that a single-atom tip in water may take a hopping motion even over a defectfree substrate, as shown in Figs. 3(a) and 3(b). Here we have assumed the tip always follows the stable trajectory. One may question the extent to which this hopping motion affects a sample's image quality. It is our hope that this 


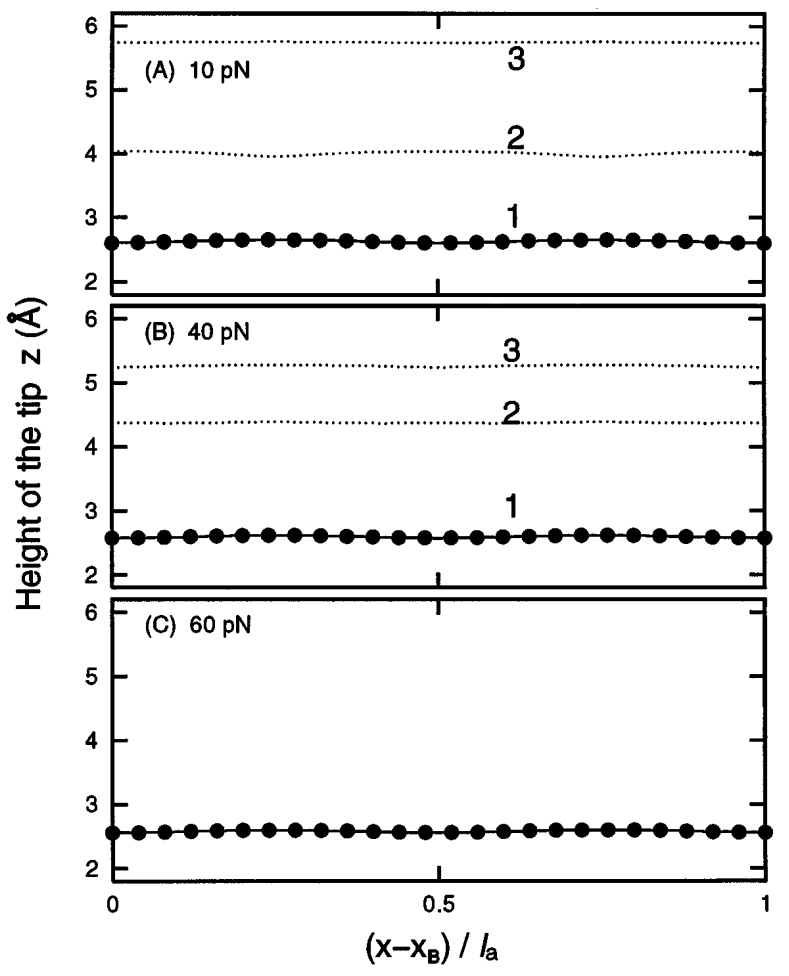

FIG. 4. The same as Fig. 3 except for the tip moving along path $B$.

theoretical work will inspire parallel AFM experiments to answer this question. Since the resolution achieved by most AFM in liquid environments so far is about one nanometer [6,7] this hopping motion may be too small to affect the AFM image. However, if an AFM is capable of true atomic resolution in liquids the hopping motion may complicate the image analysis. The newly developed carbon nanotube AFM by Smalley and coworkers seems very promising for detecting this hopping motion because the AFM tip is "sharp" on the atomic scale [27].

We are grateful to Dr. H. Tanaka for providing an original code of the RISM equation program. We also thank Dr. D. Diestler, Dr. G. Sturgeon, Dr. D. Swanson, and Dr. Y. Zhao for helpful discussions and XCZ thanks the NSF and ONR for support of this work.

[1] G. Binnig, C.F. Quate, and Ch. Gerber, Phys. Rev. Lett. 56, 930 (1986).

[2] F. Ohnesorge and G. Binnig, Science 260, 1451 (1993).

[3] J. Hu, X.D. Xiao, D. F. Ogletree, and M. Salmeron, Science 268, 267 (1995).

[4] S. J. O'Shea, M.E. Welland, and T. Rayment, Appl. Phys. Lett. 60, 2356 (1992).

[5] J.P. Cleveland, T.E. Schäffer, and P. K. Hansma, Phys. Rev. B 52, R8692 (1995).

[6] C. Bustamante and D. Keller, Phys. Today 48, 32 (1995).

[7] J. Yang, L. K. Tamm, A.P. Somlyo, and Z. Shao, J. Microsc. 171, 183 (1993).
[8] Y. L. Lyubchenko, P. I. Oden, D. Lampner, S. M. Lindsay, and K. A. Dunker, Nucl. Acids Res. 21, 1117 (1993).

[9] R. G. Horn and J. N. Israelachvili, J. Chem. Phys. 75, 1400 (1981).

[10] S. Granick, Science 253, 1374 (1991).

[11] U. Landman, W. D. Luedtke, N. A. Burnham, and R. J. Colton, Science 248, 454 (1990).

[12] L. D. Gelb and R. M. Lynden-Bell, Chem. Phys. Lett. 211, 328 (1993).

[13] Forces in Scanning Probe Methods, edited by H.-J. Güntherodt, D. Anselmetti, and E. Meyer, NATO ASI Ser. E, Vol. 286 (Kluwer Academic Publishers, Dordrecht, 1995).

[14] B. Bhushan, J. N. Israelachvili, and U. Landman, Nature (London) 374, 607 (1995).

[15] D. Chandler and H. C. Andersen, J. Chem. Phys. 57, 1930 (1972); S. J. Singer and D. Chandler, Mol. Phys. 55, 621 (1985).

[16] K. Koga, H. Tanaka, and X. C. Zeng, J. Phys. Chem. 100, 16711 (1996).

[17] W. Jorgensen, J. Am. Chem. Soc. 103, 335 (1981); B. M. Pettitt and P. J. Rossky, J. Chem. Phys. 77, 1451 (1982).

[18] S. W. Chen and P. J. Rossky, J. Phys. Chem. 97, 6078 (1993); M. Matsumoto, H. Tanaka, and K. Nakanishi, J. Chem. Phys. 99, 6935 (1993).

[19] F. Hirata, B. M. Pettitt, and P. J. Rossky, J. Chem. Phys. 77, 509 (1982).

[20] K. Koga, X. C. Zeng, and H. Tanaka, J. Chem. Phys. 106, 9781 (1997).

[21] F. Hirata and R. M. Levy, Chem. Phys. Lett. 136, 267 (1987); K. S. Schweizer and J. G. Curro, Phys. Rev. Lett. 58, 246 (1987).

[22] Larger periodic unit size is also examined and nearly the same results are obtained, indicating no appreciable size effects by using the periodic units indicated in the article.

[23] An infinite number of site-site intramolecular correlations for the tip-substrate supermolecule are treated as follows: The correlations between lattice sites of the substrate are cut off when the site-site distance $r_{i j}$ is greater than $5 l_{a}$. The correlations between the tip and lattice sites are tapered, using a switching function, in a range between $5 l_{a}-l_{a} / 2$ and $5 l_{a}+l_{a} / 2$. The correlations between the tip and all image tips lying in the surrounding unit cells are set to zero (the shortest site-site distance is $L_{y}$, which is larger than $5 l_{a}$ ). The method here is analog to the potential truncation method used in molecular dynamics simulations.

[24] D. J. Diestler, E. Rajasekaren, and X.C. Zeng, J. Phys. Chem. 101, 4992 (1997).

[25] The loads $\left(\sim 10^{-11} \mathrm{~N}\right)$ used here are comparable to typical load per atom in laboratory AFM experiments.

[26] Similar behavior has also been found in computer simulation of fluids between two parallel solid surfaces, where the surface may take hopping motion normal to the surface due to the drainage or imbibition transition of the confined fluids. See M. Schoen, D. J. Diestler, and J. H. Cushman, J. Chem. Phys. 100, 7707 (1994).

[27] H. Dai, J.H. Hafner, A. G. Rinzler, D. T. Colbert, and R.E. Smalley, Nature (London) 384, 147 (1996); D. J. Keller, Nature (London) 384, 111 (1996). 